\title{
试析化学技术在环境保护应用中存在的问题及对策
}

\author{
张俊 \\ 重庆重交再生资源开发股份有限公司 \\ DOI:10.32629/eep.v2i5.258
}

[摘 要] 在环境污染治理过程中,化学技术的应用是极其重要的。为了提高化学技术在环境保护中的应用水平,达到最佳的环 境治理效果, 必须充分了解相关化学知识, 制定与实际环境相匹配的污染治理方案, 避免滥用化学制剂导致的二次污染问题发 生,保证环境质量,提升化学技术应用的规范性与合理性。

[关键词]化学技术; 环境保护; 应用; 问题; 对策

如今, 化学技术成为环境保护中的重要技术, 但是该技 术在使用过程中也出现了一些问题, 因此只有保证化学技术 的合理应用, 才能最大限度地发挥出该技术的重要作用。鉴 于此, 本文主要分析了化学技术在环境保护中的应用与其存 在的问题, 并探讨了化学技术在环境保护应用的发展方向。

\section{1 化学技术在环境保护中的应用}

1.1 固态废物处理中的应用

在现阶段环保工作中, 城市垃圾的处理方式主要有两种, 一是无害化垃圾填埋场, 一是垃圾焚烧。前者的处理方式相 对较为简单, 不过占地面积以及资金投放效率较大, 虽说是 无害化垃圾处理, 但是产生垃圾黑液问题仍对环境有着一定 影响。后者处理方式是发达国家最常使用的一种形式, 虽然 可以将部分污染物质进行回收利用处理, 但是在应用中仍会 产生大量的资金消耗, 且燃烧后产生的废气对于空气质量有 着严重影响, 很容易导致再次污染的发生。尤其是燃烧过程 中产生的二氧化碳气体, 对于大气质量有着较为严重的影 响。基于此, 在现阶段环境保护中, 通过化学技术的应用来实 现固态废物的有效处理, 以此来降低污染物质的产生, 保证 环境质量。在使用化学技术处理中, 最常使用的方式有热分 选煤气化技术和固体废弃物电离气化技术这两种。

\section{2 大气污染处理中的应用}

大气污染的产生大多都是由于煤炭燃烧中产生的有害 物质造成的, 如一氧化碳、一氧化硫、二氧化硫等, 所以在对 大气污染进行处理时, 一般会采用脱硫法将其中含有的有害 物质释放出去。在脱硫过程中主要经历了燃前脱硫、燃中脱 硫以及燃后烟气脱硫这三个环节, 前者属于有效的预防手段, 后者则属于污染治理措施。结合目前绿色环保理念的要求, 脱硫技术在今后的发展中将成为大气污染治理的主流技术, 并起着较为重要的作用。

此外, 目前在进行煤炭脱硫技术中, 常用的是生物脱硫 法, 主要是利用生物浸出法、表面处理浮选法以及微生物絮 凝法等。还有很多研究学者把煤炭生物技术和非生物乳化技 术结合起来, 提出一种新型的煤炭脱硫技术, 生物非生物综 合技术, 这种技术可以缩短脱硫时间。在微生物菌种的基础 研究中, 国内普遍采用驯化传统菌种和利用遗传学技术, 其
主要是对微生物进行改良, 以此来降低污染气体中有害物质 的含量, 并将其转化成水等物质排出, 降低污染的产生。这些 技术的应用为我国大气污染治理带来了明显的效果。

\section{3 水污染处理中的应用}

生活污水、工业污水以及农药污染都是造成水污染问题 的主要原因。在进行水污染处理过程中, 需要坚持绿色环保 理念的相关要求, 从源头上进行污水控制和处理, 并加强各 阶段污水排放控制效率, 减少污染和浪费现象的产生, 提升 水污染处理效率, 实现水资源循环利用目标。我国在进行水 污染处理中, 最常使用的处理方式有热水锅炉雾排污技术, 该技术在使用过程中大大降低了废盐水和煮炉水的生成效 率, 提升了废水利用率, 减少了排放量。不过该技术在使用过 程中还处在初级阶段内, 仍需进一步研究和完善。

此外, 我国在农业生产工程中, 会使用很多高毒性农药, 这些农药也会对水造成严重污染。随着不断推行绿色食品的 观念, 人们对无公害绿色农药越发重视。现代使用的新型绿 色农药主要包括生物农药, 现代化学农药和光活化农药等, 这些农药可以取代此前高毒性农药的使用, 很大程度上减少 了水污染和环境污染。

\section{2 化学技术在环境保护应用中的问题}

2.1 化学技术危险性的认知不足

化学技术应用过程中涉及到一些化学物质的残留, 虽然 其对于环境污染治理有着很好的效果, 但同时也会存在一定 的副作用。而这些副作用在书本上并未进行全面系统的提及, 很多案例的存在并未充分考虑到对周边环境带来的影响, 这 导致在实践应用中很容易对环境产生影响, 使得治理过程中 又产生很多新的污染, 影响了整体治理效果, 降低了环境治 理的实效。另外, 化学技术在环境污染治理中的应用并没有 明确的理论概述作为实践上的支持, 单单依靠书本中的理论 知识, 很难提升环境治理的效果和质量, 实践应用与理论之 间存在较大偏差, 降低了化学技术的应用性。例如, 在抑制温 室效应的化学方法的处理中, 较多是对含硫物质进行脱硫操 作, 减少 $\mathrm{C} 02$ 气体的排放, 但这个过程中需要关注脱硫等具体 方法和脱硫时温度的把握这些细节部分, 掌握不清晰, 就可 能直接影响到脱硫的效果, 进而对于环境保护产生明显的负 
面影响。

\section{2 化学技术的选择较为困难}

通过将物理技术和化学技术进行比较分析可以看出, 物 理技术受到外界环境的影响相对较小, 在恶劣的工作环境也 能够按照设定好的标准要求进行操作。但是化学技术受到外 界环境的影响相对较大, 存在着较高的不稳定性, 这使得初 期制定的治理方案在落实上存在着较大差异和变动, 很容易 影响治理效果。因此, 在应用化学技术进行环境污染治理时, 除要对物质之间的化学反应进行有效掌控外, 还需要对化学 元素、离子等在环境中受到的影响进行充分分析, 只有充分 了解环境特征, 才能更好的保证实验效果, 提升治理水平, 降 低对环境及人身体带来的威胁。这就要求对化学药品的用 量、使用方法、时间等进行反复试验和检查, 进而得出较为 准确的数值参数, 保证试验效果。但是, 进行环境治理的相关 单位对于实验收到的效果存在认识不充分的情况, 没有按照 真实环境模拟治理过程, 至少课堂老师举例中没有对这方面 着重体现, 如此, 在进行环境污染问题解决的过程中久可能 出现考虑问题不全面的情况, 直接影响环境治理效果, 造成 治理效果不理想。

2.3 反应效果的掌控力度缺失

运用化学方法进行环境污染治理是一个长期艰巨的工 作, 需要工作人员做好长期和短期反应的观察工作, 以此来 更好的解决环境中存在的问题, 促进生态环境的良性发展。 但是在实际应用上, 很少会对短期效果和长期效果进行观察 与了解, 问题追踪力度也较差, 这使得一些环境问题得不到 及时解决, 进而造成了更大污染, 影响了环境保护工作的质 量和水平。

\section{3 化学技术在环境治理中应用的提升策略}

3.1 进行详细的现场环境勘察

现场环境勘察为环境治理提供了更多可靠依据, 帮助相 关人员更好的运用化学技术开展环境治理工作。只有对现场 进行详细的勘察和深入分析, 才能进一步明确污染状况、污 染源情况及产生原因, 做污染源采样工作, 进而为后续的实 验分析奠定基础。同时在实验分析过程中, 结合现场勘察数 据能够对污染物成分进行深入研究, 并运用所学的化学知识 找到正确的解决方法, 提高环境治理的水平。另外, 在现场勘 察工作中, 还需要对周边区域的环境状况进行细致分析, 充
分考虑周边环境效果, 以此来保证化学技术选用的合理性, 减少新污染物质的产生, 提升环境治理适应性。

\section{2 制定科学合理的实验方案}

由于化学技术选择的困难性, 在制定合理的治理方案前 需要进行化学实验, 以此来确定其使用效果及相关化学药品 的用量、配比情况。为此, 应建立专门的化学实验室, 便于进 行反复多次的实验验证, 进而为化学技术的可行性提供依据 和帮助。同时还应加强与政府等相关部门、机构的交流效率, 对现有资源进行整合与完善, 实现化学技术现有成果的推广 和分享, 确保化学技术能够更好的应用到环境污染治理工作 中来, 提高污染治理的水平和效果。在交流过程中, 可针对其 存在的问题进行探讨和分析, 制定合理的解决方案。而在治 理方法的确定和落实上, 则需要在实际环境中进行实验, 观 察实际工作的效果, 不能盲目的进行化学制剂的投放, 避免 出现二次污染。

3.3 加强长期效果和短期效果的观察力度

化学技术在环境保护中的应用, 短期效果是可以实时掌 控到的, 这也是为什么很多机构、部门教委关注短期效果的 原因, 但在学习化学知识时可以发现, 很多化学反应并不是 一成不变的, 是会随着环境、材料等的不同存在一定的不稳 定性, 所以在应用化学技术的过程中, 应做好长期观察的准 备, 及时对化学处理效果进行跟踪和调查, 制定合理的解决 方案, 以此来保证环境治理效果的提升。而在观察过程中, 一旦发现问题要及时进行上报和解决, 避免二次污染的产生, 降低整体治理水平提升环境保护效果。

\section{4 结语}

综上所述, 环境污染问题日益突出, 人们对于环境保护 的重视程度也在增加, 为此, 就需要结合化学技术对出现的 环境污染问题进行及时的处理, 进而对生态环境进行综合的 保护。

\section{[参考文献]}

[1]朱晓, 王涛,马春元.生物质活性炭烟气脱硫脱硝的研 究进展 [J].生物质化学工程,2018,52(02):59-66.

[2]吴兴国,韩建治.绿色化学技术在环境污染治理与保 护中的应用[J].低碳世界,2017,(07):28-29.

[3]程磊.化学技术在环境保护运用中存在的问题及对策 [J].中国新技术新产品,2016,(19):102-103. 